\title{
planificación estratégica
}

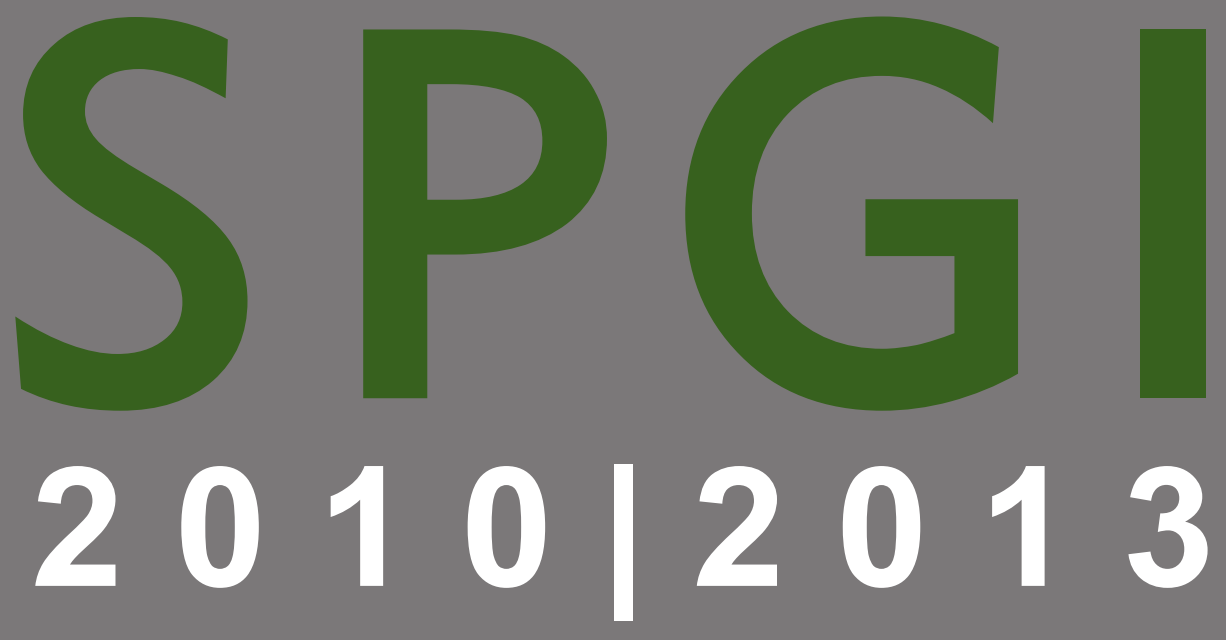

universidadnacionaldecórdoba 


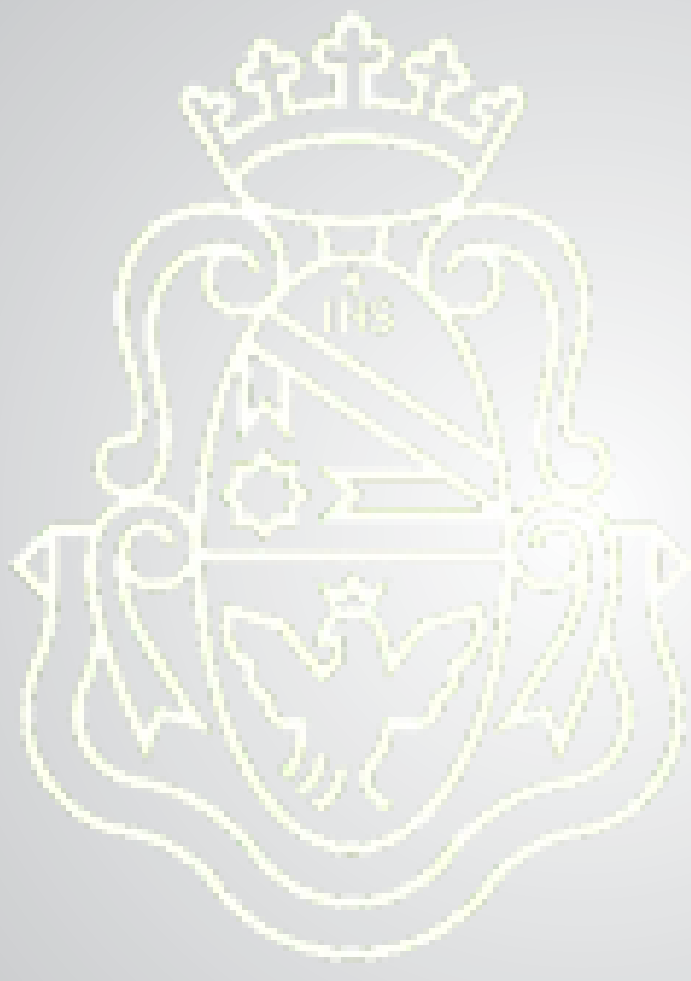

\section{planificación estratégica 2010 | 2013}

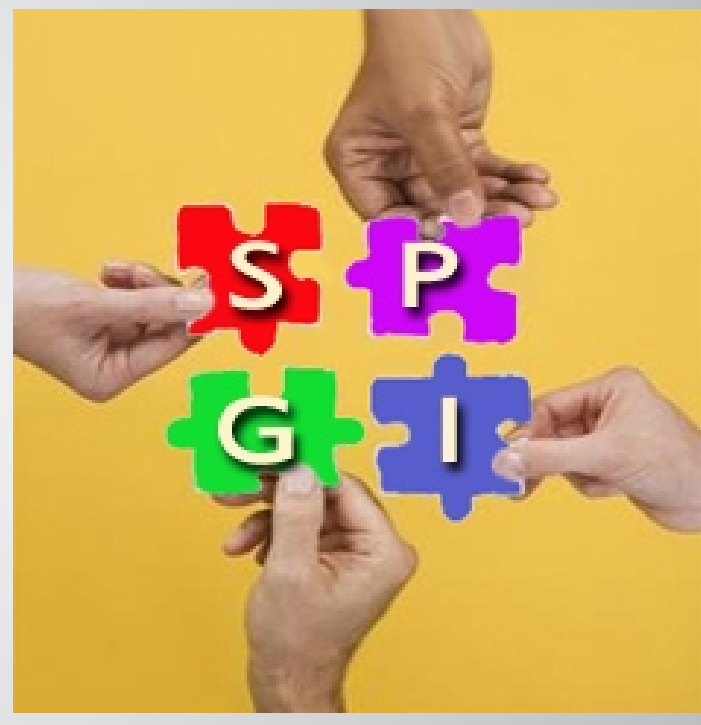

Dr. Sergio Obeide Secretario de PIan ificación y $G$ e $s t$ í $n$ Institucional 
La Secretaría de Planificación y Gestión Institucional ha formulado su planificación estratégica para el período de gestión 2010-2013. El plan resultante esta basado en una profunda revisión del rol de esta Secretaría en la Universidad y determina la misión, la visión, los ejes y objetivos concretos definidos para cada eje, todo lo cual otorga coherencia a las acciones previstas y ya iniciadas.

Cabe destacar que gran parte de los objetivos estipulados, implican una alta complejidad de ejecución dado que involucran a diversas áreas de la Secretaría de Planificación y Gestión Institucional e incluso a distintas dependencias de la Universidad, requieren aportes interdisciplinarios, demandan una precisa planificación de las actividades consecuentes y la construcción de indicadores de proceso y de resultados, así como una clara asignación de responsabilidades.

La actual estructura organizacional de la Secretaría de Planificación y Gestión Institucional no tiene la capacidad para brindar el soporte necesario a tal nivel de complejidad en el cumplimiento de los objetivos planteados, dado que se trata de una estructura funcional clásica, típica de las organizaciones administrativas, basada en una división del trabajo que genera unidades organizacionales (Direcciones Generales, Direcciones, Departamentos, etc.) a cargo de trayectos o partes acotadas de los procesos completos que son responsabilidad de la Secretaría. 
De esta manera, la estructura funcional induce a la constitución de compartimentos estancos, lo cual dificulta desarrollar a los máximos responsables una perspectiva, autoridad y responsabilidad holísticas sobre los problemas institucionales.

A modo de respuesta a estas limitaciones de la actual estructura, fue concebida y puesta en marcha una "estructura por proyectos" cuyo funcionamiento es paralelo a la estructura funcional vigente e implica nuevos roles y responsabilidades para los agentes de la Secretaría.

Adicionalmente, el personal fue capacitado en administración de proyectos, siguiendo la metodología de marco lógico. El resultado está constituido por 15 proyectos, técnicamente sólidos: antecedentes, árbol del problema, árbol de objetivos, indicadores de proceso (actividades), de resultado (productos y propósito) y de impacto (fines últimos), coordinador e integrantes formalmente designados, cronograma detallado (gráfico de Gantt). 


\section{visión}

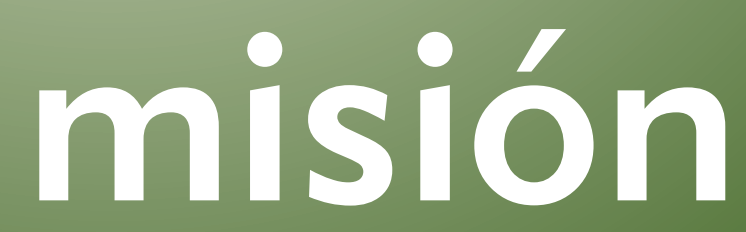




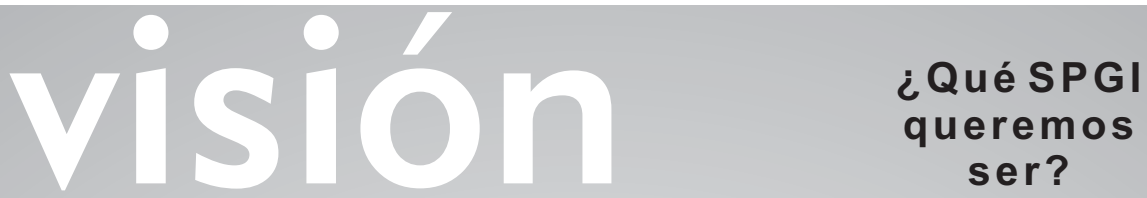

- Ser una Secretaría con niveles de excelencia en el cumplimiento de sus misiones institucionales.

- Ser una Secretaría con un alto desarrollo técnico, profesional y humano de los agentes que la integran.

- Ser una Secretaría de referencia en el ámbito interno, local y nacional.

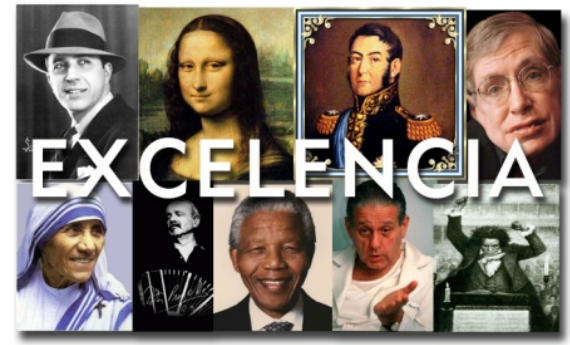

¿Cuál es la razón de ser de la SPGI?

¿Cuál es su rol

institucional?

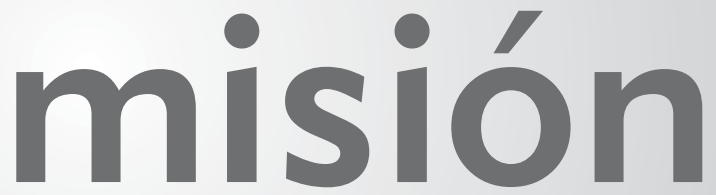

- Entender en la planificación, organización, ejecución y evaluación de los procesos económico-financieros, de personal, de desarrollo y mantenimiento de la planta física de la UNC.

- Asesorar y asistir al Sr. Rector en la formulación de políticas universitarias.

- Prestar un servicio administrativo.

- Determinar (y actualizar) para todo el ámbito de la UNC, los sistemas y procesos administrativos pertinentes.

- Asistir y asesorar en las materias de su incumbencia a las Dependencias de la UNC.

- Formular y ejecutar políticas de desarrollo de los recursos humanos no docentes de la UNC.

- Contribuir a la transparencia y publicidad de los actos de gobierno.

- Mantener activas y permanentes relaciones con los organismos externos pertinentes. 


$$
\text { propósitos }
$$

$$
\text { y ejes generales }
$$


propósito 1:administración presupuestaria Concebir la discusión presupuestaria como una instancia de planificación institucional, asignando los recursos en el marco de objetivos y políticas universitarias previamente consensuadas.

\section{propósito 2: sistemas y procesos}

Revisar los sistemas, procesos y métodos de trabajo vigentes con el objeto de lograr un incremento o mejora de los siguientes aspectos: agilidad operativa, eficacia, eficiencia, clara asignación de responsabilidades, controles necesarios, seguridad de la información manipulada, generación de información para la toma de decisiones, conciencia de prestación de un servicio por parte de los agentes involucrados.

\section{propósito 3: desarrollo de RRHH}

Reforzar el desarrollo de los Recursos Humanos No Docentes a partir de una política que atienda particularmente: los mecanismos de ingreso; el reconocimiento del mérito; la formación continua y pertinente; la revisión de las prácticas de trabajo, modos organizacionales y de liderazgo; la generación de oportunidades de promoción. 
propósito 4: interrelación con las dependencias

Ejercer una administración en permanente contacto y con activa participación de las Unidades Académicas y demás Dependencias en el tratamiento de los temas que competen a la SPGI.

propósito 5: transparencia

Contribuir a la transparencia de los actos administrativos y de gobierno, a partir de la difusión de información pública y pertinente.

propósito 6: infraestructura

Propiciar una política de desarrollo y mantenimiento de la infraestructura universitaria que responda a una clara y equitativa lectura de las necesidades institucionales.

propósito 7: relaciones externas Participar activamente en los ámbitos externos que correspondan (particularmente el CIN, la SPU, el Congreso de la Nación) para aportar a la formulación de políticas nacionales de educación superior; para explicar, ilustrar y reclamar por las necesidades presupuestarias de la UNC; para atender todo otro aspecto que se entienda pertinente al ámbito de la SPGI. 


$$
\begin{aligned}
& \text { acciones } \\
& \text { realizadas } \\
& 2007 \quad 2010
\end{aligned}
$$




\section{propósito 1 :administración presupuestaria}

- Desarrollo de un modelo de asig. de recursos basado en pautas objetivas.

- Res. HCS 159/08: Creación Fondo de Antigüedad y Salario Familiar.

- Res. HCS 160/08: Creación Fondo de Recursos Humanos Académicos.

- Res. HCS 403/08: Asignación presupuestaria de Inc. 1, F11, aplicando el modelo de asignación de recursos.

- Res. HCS 681/08: Compensación de déficits históricos y establecimiento de pautas de compensación de eventuales déficits futuros.

- Inicio del desarrollo de un sistema de información presupuestaria para las Dependencias.

- Generación de nuevas fuentes de recursos propios (Bancos) y revisión de la eficiencia del gasto (EPEC, telefonía IP, seguros, ART, etc.). 


\section{propósito 2: sistemas y procesos}

- Puesta en marcha de PILAGÁ.

- Aplicación completa de PAMPA.

- Puesta en marcha de COMDOC.

- Ord. HCS 15/08: Descentralización de contrataciones de personal.

- Ord. HCS 18/08: Descentralización de convenios específicos.

- Desarrollo y puesta en marcha del Sistema de Registro Integral.

- Análisis y evaluación para la aplicación de banca electrónica. Primeros pasos de aplicación.

\section{propósito 3: desarrollo de RRHH}

- Puesta en marcha de la Tecnicatura en Adm. Universitaria.

- Programa de Capacitación No Docente.

- Programa de Formación de Adultos.

- Programa de Mejora Continua (PMC).

- Nuevas normas internas:

- Condiciones de ingreso y promoción

- Límites a las contrataciones

- Licencias

- Adicionales y suplementos

- Plan de concursos SPGI.

- Análisis organizacional SPGI.

propósito 4: interrelación con las dependencias

- Creación del Portal de la SPGI.

- Pasos iniciales en la creación de redes temáticas y foros (Presupuesto, Contrataciones, Unidad de Evaluación de Actividades de Formación, ART).

- Convocatorias varias a reuniones para el tratamiento de diversos temas.

acciones realizadas 2007 | 2010 


\section{propósito 4: interrelación con la dependencias}

- Creación del Portal de la SPGI.

- Pasos iniciales en la creación de redes temáticas y foros (Presupuesto, Contrataciones, Unidad de Evaluación de Actividades de Formación, ART).

- Convocatorias varias a reuniones para el tratamiento de diversos temas.

\section{propósito 5: transparencia}

- No constituyó un propósito-eje del periodo de gestión 2007 . 2010.

\section{propósito 6: infraestructura}

- Creación del Banco de Proyectos de Obras de la UNC.

- Plan Trianual de Obras:

- Equilibrio entre nueva obra y mantenimiento.

- Concepción integral del campus.

- Puesta en valor de los espacios de uso común (plazas, corredores, iluminación, mantenimiento de espacios verdes, recolección de residuos).

- Plan integral de desarrollo y crecimiento.

\section{propósito 7: relaciones externas}

- Integrante de la Comisión de Asuntos Económicos del CIN.

- Integrante del GEPP (Grupo Ejecutivo de Pautas

Presupuestarias del CIN).

- Integrante de la Comisión de Enlace CIN - SPU.

- Diálogo técnico permanente con el Consorcio SIU. 


$$
\text { objetivos }
$$




\section{propósito 1:administración presupuestaria}

- Revisión del Modelo de reasignación de recursos.

- Sistema de información para la toma de decisiciones

-Planta y ejecución del inciso 1: 03.

- Rediseño de la Red Programática y Plan de Cuentas.

- Tablero de control.

propósito 2: sistemas y procesos

- Módulo Cliente Contrataciones (MCC)? / Sistema SIUDiaguitas?.

- Modificaciones y plena utilización del Sistema de Registro de Contrataciones.

- Certificación electrónica de servicios.

- Módulo web de administración económico-financiera de las Dependencias.

- Banca electrónica.

- Revisión y rediseño de procedimien tos claves.

- Revisión y rediseño del proceso de cierre de ejercicio. 


\section{propósito 3:desarrollo de RRHH}

- Cumplir con los planes de trabajo aprobados para los Programas de Capacitación (presencial), de Formación de Adultos y de Mejora Continua.

- Puesta en marcha del Programa de Capacitación (modalidad a distancia).

- Puesta en marcha del Programa de Orientación Institucional.

- Sistematización del Programa de Mejora Continua.

- Organización interna de la SPGI (Fase 2).

Otros objetivos contemplados en la plataforma de gobierno, surgidos de las discusiones en comisión de la comunidad universitaria, y cuya resolución no depende enteramente de la SPGI:

- "Sistema de evaluación de resultados del personal No Docente".

- "Elaborar esquemas organizacionales que definan áreas y jerarquías generales comunes a todas las Dependencias".

- "Revisar el Régimen de Concursos y promover su realización en todas las Dependencias". 
propósito 4: interrelación con las dependencias

- Constituir redes por áreas y/o temas específicos: presupuesto, contrataciones, sueldos, informática, contabilidad, etc.

- Convocar a reuniones generales con agendas concretas de trabajo.

- Realizar visitas a las Dependencias con fines diagnósticos y de asesoría.

- Producir un salto cualitativo del Portal de la SPGI: acceso desde el Portal UNC; revisión del mapa del sitio; contenidos y estética; actualización distribuida; etc.

- Revisión de las misiones y funciones del CIRI.

\section{propósito 5: transparencia}

- Difundir por diversos medios (Portal SPGI, publicaciones específicas, comunicaciones, etc.) información pública, particularmente referida a:

-Administración presupuestaria.

- Contrataciones.

- Obra pública.

- Otros. 


\section{propósito 6: infraestructura}

- Culminar la ejecución del Plan Trianual de Obras 2008 2010.

- Elaboración de un nuevo plan de obras.

- Revisar y replantear las responsabilidades asignadas al nivel central y a las dependencias en lo relativo al mantenimiento edilicio.

- Revisar y replantear la organización de la administración central para la atención de sus roles actuales.

propósito 7: relaciones externas

- Mantener presencia y representación institucional en los espacios conseguidos (particularmente CIN y SPU).

- Dar cumplimiento a la agenda técnica y política acordada en el CIN.

- Mantener los contactos necesarios con el Consorcio SIU para el cumplimiento de la agenda de la SPGI. 


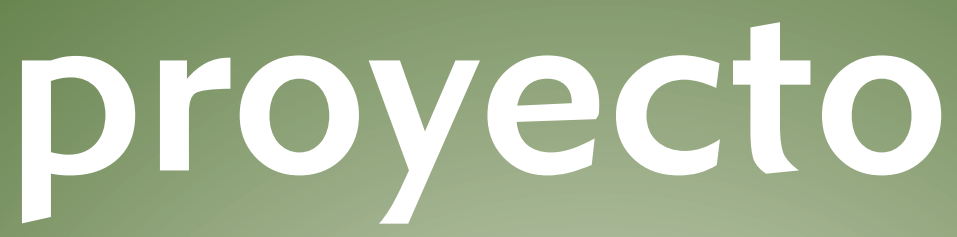

Es la organización y programación del conjunto de actividades requeridas para la consecución de un objetivo.

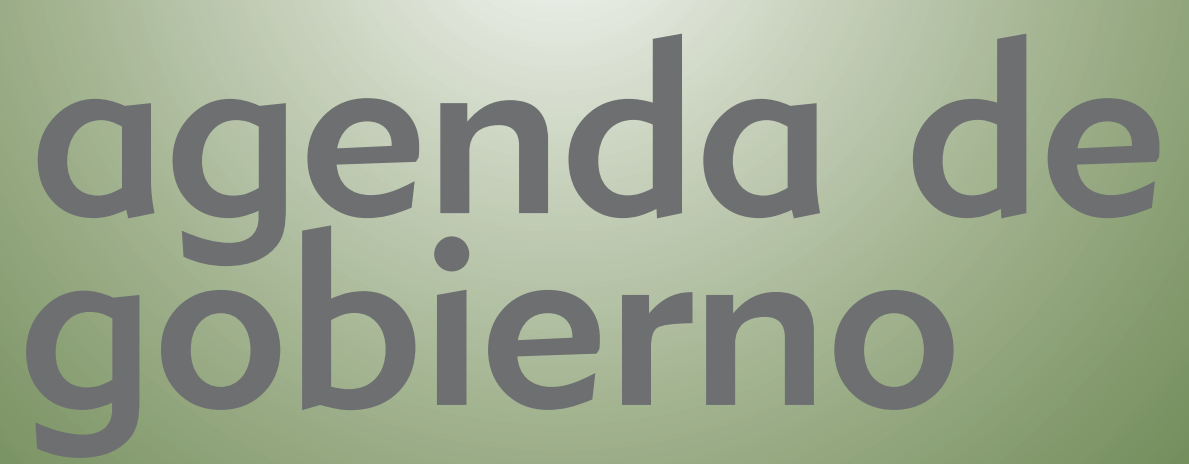

Es el conjunto de problemas, objetivos y proyectos que han sido priorizados para su tratamiento y ejecución en un periodo de gobierno. 


\section{organización para la ejecución de la agenda}

Situación actual: estructura funcional SPGI

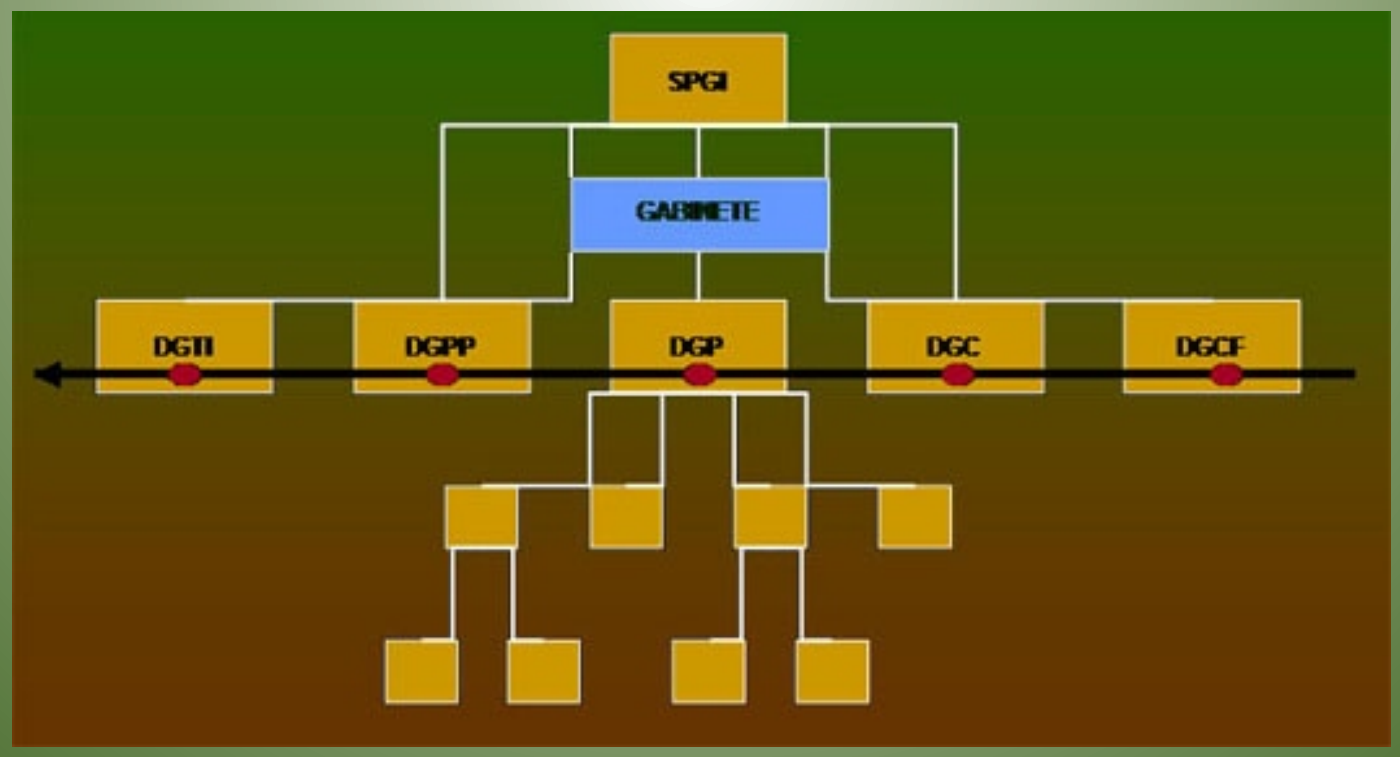




\section{nueva}

organización

para la ejecución

de la agenda

estructura por proyectos superpuesta a la estructura funcional

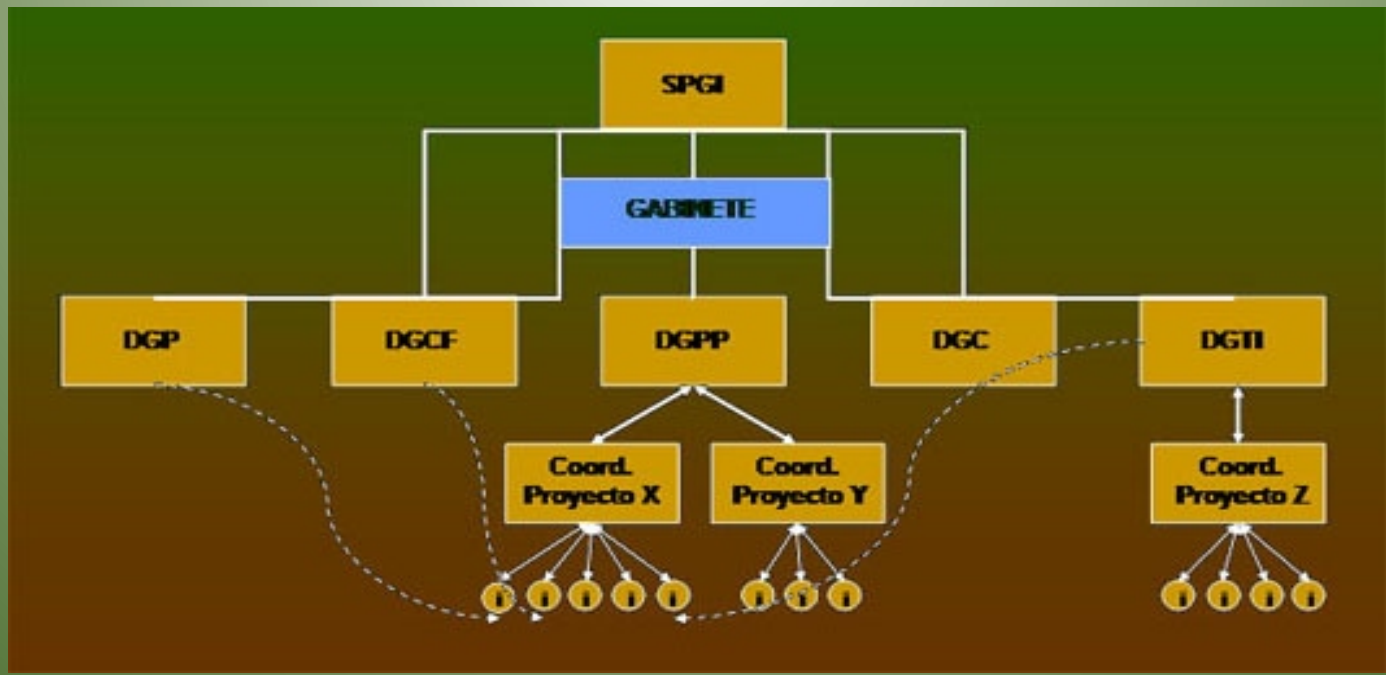




\section{roles y \\ responsa \\ bilidades \\ asociados \\ a la estructura por proyectos}




\section{coordinador de proyecto}

- Conducir el proceso de consultas y discusiones tendientes a la elaboración del proyecto.

- Realizar la redacción final del proyecto según las pautas establecidas por la Secretaría.

- Coordinar las actividades conducentes al cumplimiento de los objetivos del proyecto en los plazos previstos.

- Elaborar los informes de avance requeridos y el informe final.

\section{director general}

- Participar activamente, en calidad de integrante, del proceso tendiente a la elaboración de los proyectos que hayan sido asignados bajo su órbita.

- Intervenir en la ejecución de los proyectos, en calidad de integrante, en aquellos casos en que así se haya contemplado.

- Facilitar la tarea de los coordinadores a efectos de construir la viabilidad de la ejecución de los proyectos.

- Efectuar el seguimiento y monitoreo permanente de los niveles de ejecución de los proyectos bajo su órbita. 


$$
\begin{aligned}
& \text { conside } \\
& \text { raciones } \\
& \text { fthales }
\end{aligned}
$$




\section{Los siguientes actos administrativos}

\section{serán formalizados por medio de}

\section{Resolución SPGI:}

- Designación de los Coordinadores e Integrantes de cada

Proyecto y responsabilidades asignadas.

- Aprobación de los proyectos elaborados (documentos).

- Asignación de proyectos a la órbita de cada Dirección

General y responsabilidades asociadas de los DG.

Las Resoluciones SPGI indicadas, así como los informes de avance e informes finales, serán colocados en el portal de la SPGI.

Instrumentación de mecanismos para el reconocimiento formal del mérito. 


\section{Lineamientos de acción 2010 | 2013 Junio 2010}

